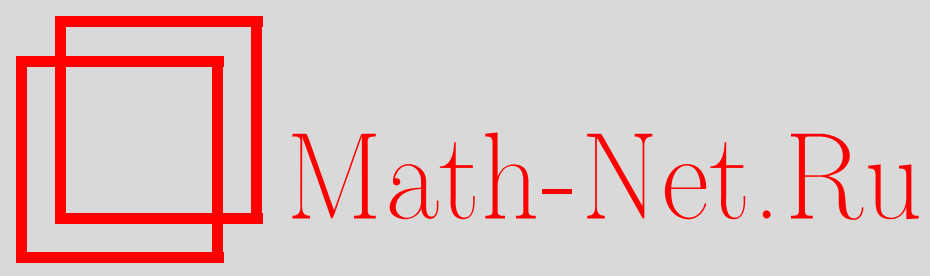

Г. Г. Ониани, О возможных значениях верхней и нижней производных, Матем. заметки, 1998, том 64, выпуск 1, 107-114

DOI: https://doi.org/10.4213/mzm1376

Использование Общероссийского математического портала Math-Net.Ru подразумевает, что вы прочитали и согласны с пользовательским соглашением http://www . mathnet.ru/rus/agreement

Параметры загрузки:

IP : 54.172 .240 .79

26 апреля 2023 г., 18:21:57

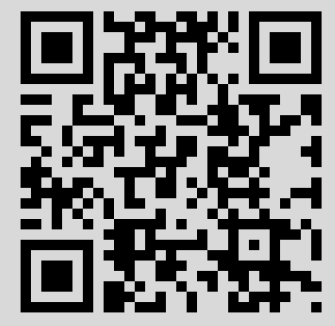




\section{О ВОЗМОЖНЫХ ЗНАЧЕНИЯХ ВЕРХНЕЙ И НИЖНЕЙ ПРОИЗВОДНЫХ}

\section{Г.Г. Ониани}

В работе изучено поведение верхней и нижней производных интегралов относительно дифференциальных базисов, образованных из интервалов. Основной результат состоит в том, что аналог известной теоремы Безиковича, вообще говоря, не имеет места для инвариантных относительно сдвига буземан-феллеровских базисов, образованных из интервалов.

Библиография: 2 названия.

1. Определения и обозначения. Отображение $B$, определенное на $\mathbb{R}^{n}$, называется дифференииальныцм базисомв в $\mathbb{R}^{n}$, если для каждого $x \in \mathbb{R}^{n} B(x)$ является семейством открытых ограниченных множеств, содержаших точку $x$, таких, что существует последовательность

$$
\left\{R_{k}\right\} \subset B(x), \quad \operatorname{diam} R_{k} \rightarrow 0, \quad k \rightarrow \infty .
$$

Для $f \in L_{\mathrm{loc}}\left(\mathbb{R}^{n}\right)$ числа

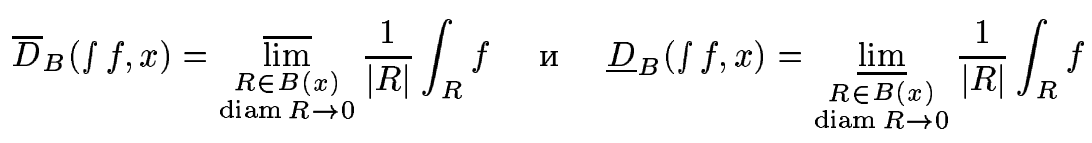

называют соответственно верхней и нижней производными интеграла от $f$ в точке $x$. Если верхняя и нижняя производные совпадают, то их общее значение называют производной от интеграла от $f$ в точке $x$ и обозначают через $D_{B}\left(\int f, x\right)$. Говорят, что базис $B$ дифференцирует интеграл от $f$, если для почти всех $x D_{B}\left(\int f, x\right)=f(x)$.

Базис $B$ называют подбазисом $B^{\prime}$ (запись: $\left.B \subset B^{\prime}\right)$ ), если $B(x) \subset B^{\prime}(x), x \in \mathbb{R}^{n}$. Базис $B$ называют базисом Буземана-Феллера (ВF-базисом), если для каждого $R \in \bar{B}=$ $\bigcup_{x \in \mathbb{R}^{n}} B(x)$ имеем $R \in B(y)$ для всякого $y \in R$. ВF-базис $B$ называется инвариантны.м относительно гомотетии (НІ-базисом), если для каждого $R \in \bar{B}$ в $\bar{B}$ входит любое множество, гомотетичное $R$. Базис $B$ называется инвариантным относительно сдвига (ТІ-базисом), если $B(x)=\{x+R: R \in B(0)\}, x \in \mathbb{R}^{n}$, где 0 - начало координат в $\mathbb{R}^{n}$.

Через $B_{2}$ будем обозначать базис в $\mathbb{R}^{2}$, для которого $B(x)$ состоит из всех двумерных интервалов, содержащих точку $x, x \in \mathbb{R}^{2}$.

Условимся, что $I^{n}=(0,1)^{n}$ и $f \in L\left(I^{n}\right)$, если $f \in L\left(\mathbb{R}^{n}\right)$ и $\operatorname{supp} f \subset I^{n}$. 
2. Основные результаты. Согласно известной теореме Безиковича (см. [1] или $[2$, c. 96]) о возможных значениях верхней и нижней производных для каждой функции $f \in L\left(\mathbb{R}^{2}\right)$ оба множества

$$
\left\{x \in \mathbb{R}^{2}: f(x)<\bar{D}_{B_{2}}\left(\int f, x\right)<\infty\right\}, \quad\left\{x \in \mathbb{R}^{2}:-\infty<\underline{D}_{B_{2}}\left(\int f, x\right)<f(x)\right\}
$$

имеют меру 0.

Гусманом и Менаргесом (см. [2, с. 100]) было установлено некоторое обобщение этого результата. Из их теоремы следует, что аналог утверждения Безиковича справедлив для инвариантных относительно гомотетии ВF-базисов $B \subset B_{2}$.

Возникает вопрос: можно ли теорему Безиковича распространить на инвариантные относительно сдвига ВF-базисы $B \subset B_{2}$ ?

Отрицательный ответ на этот вопрос дает следующая

Tеорема 1. Существует инвариантный относительно сдвига ВF-базис ВС $B_{2}$, для которого найдется функиия $f \in L\left(I^{2}\right), f \geqslant 0$, такая, что $f(x)<\bar{D}_{B}\left(\int f, x\right)<\infty$ почти всюду на $I^{2}$.

Нетрудно доказать, что (см. замечание 1 ) если $B \subset B_{2}$-инвариантньй относительно сдвига базис, то для каждой $f \in L\left(\mathbb{R}^{2}\right)$ почти всюду на $\mathbb{R}^{2}$ выполняются неравенства

$$
\underline{D}_{B}\left(\int f, x\right) \leqslant f(x) \leqslant \bar{D}_{B}\left(\int f, x\right) .
$$

Возникает вопрос: справедлив ли аналогичный факт для произвольных базисов $B \subset B_{2}$ ? Отрицательньй ответ дает

Теорема 2. Существует ВF-базис $B \subset B_{2}$, для которого найдется функиия $f \in L\left(I^{2}\right), f \geqslant 0$, такая, что

$$
f(x)<\underline{D}_{B}\left(\int f, x\right)=\infty
$$

почти всюду на $I^{2}$.

3. Доказательства теорем. Для интервала $I$ и $h>0$ через $h I$ будем обозначать интервал $H(I)$, где $H$ - гомотетия с центром в центре $I$ с коэффищиентом $h$.

Для интервала $I$ и $h>1$ через $M(I, h)$ обозначим объединение всех интервалов $J$ со свойствами: $J \supset I$ и $|J|=h|I|$. Множество $M(I, h)$ называют лестничей Бора. Отметим некоторые свойства $M(I, h)$ :

$$
M(I, h) \subset(2 h+1) I, \quad|M(I, h)|>h \ln h|I| .
$$

ДокАЗАТЕЛЬСТВо ТЕОРЕМЫ 1. Пусть $h_{k} \uparrow \infty, h_{k}>1,-$ последовательность такая, что

$$
\sum_{k=1}^{\infty} \frac{1}{h_{k}}<\infty, \quad \sum_{k=1}^{\infty} \frac{\ln h_{k}}{h_{k}}=\infty .
$$

Допустим $n_{k}=2^{m_{k}}$, где $m_{k} \in \mathbb{N}, k \in \mathbb{N}$ и и $n_{k} \uparrow \infty$. Для каждого $k$ разобьем $I^{2}$ на $n_{k}^{2}$ равных квадратных интервалов и полученные квадраты обозначим через $I_{k, n}^{2}$, а длину их сторон - через $\Delta_{k}$. Для каждого $I_{k, n}^{2}$ рассмотрим квадратньй интервал $I_{k, n}$ 
с центром в центре $I_{k, n}^{2}$ со стороной длины $\delta_{k}=\Delta_{k} /\left(2 h_{k}+1\right)$. Будем считать $n_{k} \uparrow \infty$ так, что $\delta_{k}>\Delta_{k+1}, k \in \mathbb{N}$.

Согласно (1) при $k \in \mathbb{N}, n=1, \ldots, n_{k}^{2}$

$$
M\left(I_{k, n}, h_{k}\right) \subset\left(2 h_{k}+1\right) I_{k, n}=I_{k, n}^{2}, \quad\left|M\left(I_{k, n}, h_{k}\right)\right|>h_{k} \ln h_{k}\left|I_{k, n}\right| .
$$

Следовательно, при $k \in \mathbb{N}$

$$
\begin{aligned}
\left|\bigcup_{n=1}^{n_{k}^{2}} M\left(I_{k, n}, h_{k}\right)\right| & =\sum_{n=1}^{n_{k}^{2}}\left|M\left(I_{k, n}, h_{k}\right)\right| \\
& \geqslant \sum_{n=1}^{n_{k}^{2}} h_{k} \ln h_{k}\left|I_{k, n}\right|=\sum_{n=1}^{n_{k}^{2}} h_{k} \ln h_{k} \frac{\left|I_{k, n}^{2}\right|}{\left(2 h_{k}+1\right)^{2}}>\frac{\ln h_{k}}{9 h_{k}} .
\end{aligned}
$$

Для каждого $k \in \mathbb{N}$ рассмотрим те интервалы $I_{k+1, n}^{2}, n=1, \ldots, n_{k+1}^{2}$, которые входят в $\bigcup_{n=1}^{n_{k}^{2}} M\left(I_{k, n}, h_{k}\right)$, и их объединение обозначим через $O_{k}$. Легко видеть, что если $n_{k}$ достаточно быстро стремится к $\infty$, то

$$
\left|O_{k}\right| \geqslant \frac{1}{2}\left|\bigcup_{n=1}^{n_{k}^{2}} M\left(I_{k, n}, h_{k}\right)\right|, \quad k \in \mathbb{N} .
$$

Будем считать, что $\left\{n_{k}\right\}$ подобрано так, что вьполняется (4). Используя свойства двоично-рациональных интервалов, легко убедиться в независимости последовательности $\left\{O_{k}\right\}$, т.е. для каждого $m \in \mathbb{N}$ и для различных натуральных чисел $k_{1}, \ldots, k_{m}$

$$
\left|\bigcap_{j=1}^{m} O_{k_{j}}\right|=\prod_{j=1}^{m}\left|O_{k_{j}}\right|
$$

В силу (2)-(4) вьполнено $\sum_{k=1}^{\infty}\left|O_{k}\right|=\infty$. Следовательно, используя лемму Бореля-Кантелли, заключаем, что $\lim _{k \rightarrow \infty} O_{k}$ имеет полную меру в $I^{2}$. Отсюда в силу включения

$$
O_{k} \subset \bigcup_{n=1}^{n_{k}^{2}} M\left(I_{k, n}, h_{k}\right)
$$

получаем

$$
\varlimsup_{k \rightarrow \infty} \bigcup_{n=1}^{n_{k}^{2}} M\left(I_{k, n}, h_{k}\right) \text { имеет полную меру в } I^{2} \text {. }
$$

Пусть $G_{k}, k \in \mathbb{N},-$ семейство всех интервалов со сторонами длины не меньше чем $\delta_{k}$, с площадями равными $h_{k} \delta_{k}^{2}$.

Базис $B$ определим следуюшим образом:

$$
B(x)=\left\{I \in B_{2}(x): I \in \bigcup_{k=1}^{\infty} G_{k}\right\}, \quad x \in \mathbb{R}^{2}
$$


Очевидно, $B \subset B_{2}$ и $B$ является BF- и ТІ-базисом.

Пусть

$$
f_{k}=\sup _{n=1, \ldots, n_{k}^{2}} h_{k} \chi_{I_{k, n}}, \quad k \in \mathbb{N}, \quad \text { и } \quad f=\sum_{k=1}^{\infty} f_{k} .
$$

Очевидно, $\operatorname{supp} f \subset I^{2}$ и $f \geqslant 0$. Легко видеть, что при $k \in \mathbb{N}$

$$
\left\|f_{k}\right\|_{1}=h_{k}\left|\bigcup_{n=1}^{n_{k}^{2}} I_{k, n}\right|=h_{k} \sum_{n=1}^{n_{k}^{2}} \frac{1}{\left(2 h_{k}+1\right)^{2}}\left|I_{k, n}^{2}\right|<\frac{1}{h_{k}},
$$

откуда согласно (2)

$$
\sum_{k=1}^{\infty}\left\|f_{k}\right\|_{1}<\sum_{k=1}^{\infty} \frac{1}{h_{k}}<\infty
$$

означает, что $f \in L\left(I^{2}\right)$.

Легко видеть, что

$$
\sum_{k=1}^{\infty}\left|\bigcup_{n=1}^{n_{k}^{2}} I_{k, n}\right|<\infty
$$

Следовательно,

$$
\left|\varlimsup_{k \rightarrow \infty} \bigcup_{n=1}^{n_{k}^{2}} 3 I_{k, n}\right|=0 .
$$

Очевидно, $B$ дифференцирует $\int f, k \in \mathbb{N}$, т.е. множество $A_{k}, k \in \mathbb{N}$, состоящее из всех точек $x$, для которых $D_{B}\left(\int f_{k}, x\right)=f_{k}(x)$, имеет полную меру в $\mathbb{R}^{2}$.

Обозначим

$$
A=\left(\bigcap_{k=1}^{\infty} A_{k} \cap \varlimsup_{k \rightarrow \infty} \bigcup_{n=1}^{n_{k}^{2}} M\left(I_{k, n}, h_{k}\right)\right) \backslash \varlimsup_{k \rightarrow \infty} \bigcup_{n=1}^{n_{k}^{2}} 3 I_{k, n} .
$$

В силу сказанного вьше и (5) $A$ имеет полную меру в $I^{2}$.

Пусть $x \in A$. Ясно, что найдется $k_{x} \in \mathbb{N}$, для которого

$$
x \notin \bigcup_{n=1}^{n_{k}^{2}} 3 I_{k, n} \quad \text { при } \quad k>k_{x} .
$$

Очевидно, при $\operatorname{diam} I \rightarrow 0, I \in B(x)$, имеем

$$
\frac{1}{|I|} \int_{I} \sum_{k=1}^{k_{x}} f_{k} \rightarrow \sum_{k=1}^{k_{x}} f_{k}(x)=f(x) .
$$

Допустим $I \in B(x)$ и $I \in \bigcup_{k \geqslant k_{x}+2} G_{k}$. Через $k_{I}$ обозначим число, для которого $I \in G_{k_{I}}$. Легко проверить, что $\operatorname{diam} I<2 h_{k_{I}} \delta_{k_{I}}<\Delta_{k_{I}}$. Следовательно, так как 
$\delta_{k}>\Delta_{k+1}, k \in \mathbb{N}$, имеем $\operatorname{diam} I<\delta_{k}$ при $k_{x}<k<k_{I}$. Отсюда в силу (6) при $k_{x}<k<k_{I}$

$$
I \cap \bigcup_{n=1}^{n_{k}^{2}} I_{k, n}=\varnothing
$$

Стало быть,

$$
\int_{I} f_{k}=0 \quad \text { при } \quad k_{x}<k<k_{I} .
$$

Имеем $I \in G_{k_{I}}$, значит $I$ может пересекать только один из квадратов $I_{k, n}, n=1, \ldots, n_{k_{I}}^{2}$. Следовательно, так как $|I|=h_{k_{I}} \delta_{k_{I}}^{2}$, получаем

$$
\frac{1}{|I|} \int_{I} f_{k_{I}} \leqslant \frac{1}{h_{k_{I}} \delta_{k_{I}}^{2}} h_{k_{I}} \delta_{k_{I}}^{2}=1
$$

Докажем, что при $k>k_{I}$

$$
\left|I \cap \bigcup_{n=1}^{n_{k}^{2}} I_{k, n}\right| \leqslant 9|I| \cdot\left|\bigcup_{n=1}^{n_{k}^{2}} I_{k, n}\right| .
$$

Для доказательства этого достаточно отметить следующие легко проверяемые факты:

1) $d_{I}>\Delta_{k}$ при $k>k_{I}\left(d_{I}\right.$ - длина меньшей стороны интервала $\left.I\right)$;

2) пусть $P_{k}, k \in \mathbb{N},-$ множество вершин квадратов $I_{k, n}^{2}, n=1, \ldots, n_{k}^{2}$; если вершины интервала $J \subset I^{2}$ принадлежат множеству $P_{k}$, то

$$
\left|J \cap \bigcup_{n=1}^{n_{k}^{2}} I_{k, n}\right| \leqslant|J| \cdot\left|\bigcup_{n=1}^{n_{k}^{2}} I_{k, n}\right|
$$

3) пусть $k \in \mathbb{N}$; для каждого интервала $J \subset I^{2}, d_{J} \geqslant \Delta_{k}$, найдется интервал $J^{\prime} \supset J,\left|J^{\prime}\right| \leqslant 9|J|$, с вершинами, принадлежащими $P_{k}$.

Из (10) вытекает, что при $k>k_{I}$

$$
\int_{I} f_{k}=h_{k}\left|I \cap \bigcup_{n=1}^{n_{k}^{2}} I_{k, n}\right| \leqslant 9 h_{k}|I| \cdot\left|\bigcup_{n=1}^{n_{k}^{2}} I_{k, n}\right|=9|I| \cdot\left\|f_{k}\right\|_{1} .
$$

Следовательно,

$$
\frac{1}{|I|} \int_{I} \sum_{k>k_{I}} f_{k}=\sum_{k>k_{I}} \frac{1}{|I|} \int_{I} f_{k} \leqslant 9 \sum_{k>k_{I}}\left\|f_{k}\right\|_{1} \leqslant 9\|f\|_{1} .
$$

Из (7)-(9) и (11) легко заключаем, что

$$
\bar{D}_{B}\left(\int f, x\right) \leqslant f(x)+1+9\|f\|_{1}, \quad x \in A .
$$


Поэтому найдется последовательность $k_{j} \uparrow \infty$, для которой

$$
x \in \bigcup_{n=1}^{n_{k_{j}}^{2}} M\left(I_{k_{j}, n}, h_{k_{j}}\right), \quad j \in \mathbb{N}
$$

В силу определения базиса $B$ и множества $M(I, h)$ при каждом $j$ найдется $I_{j} \in B(x)$ такой, что

$$
\frac{1}{\left|I_{j}\right|} \int_{I_{j}} f_{k_{j}}=1, \quad \operatorname{diam} I_{j}<\Delta_{k_{j}}
$$

Из (7) и (12) непосредственно получаем

$$
\bar{D}_{B}\left(\int f, x\right) \geqslant f(x)+1 \text {. }
$$

Теперь, учитьвая, что $A$ имеет полную меру в $I^{2}$, в силу произвольности $x \in A$ в неравенствах (11) и (13) выполнено

$$
f(x)+1 \leqslant \bar{D}_{B}\left(\int f, x\right) \leqslant f(x)+1+9\|f\|_{1}
$$

почти всюду на $I^{2}$. Теорема 1 доказана.

ДОКАЗАТЕЛЬСТВО ТЕОРЕМЫ 2. Пусть $h_{k}>k, k \in \mathbb{N}$, и

$$
\sum_{k=1}^{\infty} \frac{k}{\ln \left(h_{k} / k\right)}<\infty
$$

Пусть $Q$ - некоторьй квадратньй интервал. Для каждого $k \in \mathbb{N}$ покроем почти весь $I^{2}$ последовательностью попарно пересекающихся множеств, которые гомотетичны $M\left(Q, h_{k} / k\right)$ и имеют диаметры меньше чем $1 / k$. Положим $Q_{k, n}=H_{k, n}(Q), k, n \in \mathbb{N}$, где $H_{k, n}$ - гомотетия, переводящая $M\left(Q, h_{k} / k\right)$ в $n$-й член указанной последовательности (которьй, очевидно, равен $\left.M\left(Q_{k, n}, h_{k} / k\right)\right)$.

Пусть

$$
f_{k}=\sup _{n \in \mathbb{N}} h_{k} \chi_{Q_{k, n}}, \quad k \in \mathbb{N}, \quad \text { и } \quad f=\sum_{k=1}^{\infty} f_{k} .
$$

Очевидно, $f \geqslant 0$ и $\operatorname{supp} f \subset I^{2}$. Так как в силу (1) при $k, n \in \mathbb{N}$

$$
\left|M\left(Q_{k, n}, \frac{h_{k}}{k}\right)\right|>\frac{h_{k}}{k} \ln \frac{h_{k}}{k}\left|Q_{k, n}\right|,
$$

TO

$$
\left\|f_{k}\right\|_{1}=\sum_{n=1}^{\infty} h_{k}\left|Q_{k, n}\right|<\sum_{n=1}^{\infty} \frac{k}{\ln \left(h_{k} / k\right)}\left|M\left(Q_{k, n}, \frac{h_{k}}{k}\right)\right|=\frac{k}{\ln \left(h_{k} / k\right)} .
$$

Следовательно, из (14) заключаем, что $\sum_{k=1}^{\infty}\left\|f_{k}\right\|_{1}<\infty$, значит $f \in L\left(I^{2}\right)$.

Обозначим

$$
T_{k, n}=\left\{I \in \bar{B}_{2}: I \supset Q_{k, n},|I|=\frac{h_{k}}{k}\left|Q_{k, n}\right|\right\}, T_{k}=\bigcup_{n=1}^{\infty} T_{k, n}, \quad k, n \in \mathbb{N}
$$


Легко видеть, что при $k \in \mathbb{N}$ и $I \in T_{k}$

$$
\frac{1}{|I|} \int_{I} f_{k}=k
$$

По построению множество

$$
A=\bigcap_{k=1}^{\infty} \bigcup_{I \in T_{k}} I=\bigcap_{k=1}^{\infty} \bigcup_{n=1}^{\infty} M\left(Q_{k, n}, \frac{h_{k}}{k}\right)
$$

имеет полную меру в $I^{2}$. Пусть при каждом $k \in \mathbb{N} G_{k} \subset I^{2}$ - открытое множество такое, что $I^{2} \backslash A \subset G_{k}$ и $\left|G_{k}\right|<1 / 2^{k}$. При всяком $k \in \mathbb{N}$ каждому $x \in I^{2} \backslash A$ сопоставим интервал $I_{k}(x) \ni x, I_{k}(x) \subset G_{k}, \operatorname{diam} I_{k}(x)=\operatorname{dist}\left(x, \partial G_{k}\right) / k\left(\partial G_{k}-\right.$ граница $\left.G_{k}\right)$. Введем обозначения

$$
R_{1}=\bigcup_{k=1}^{\infty} T_{k}, \quad R_{2}=\left\{I_{k}(x): x \in I^{2} \backslash A, k \in \mathbb{N}\right\}, \quad R_{3}=\bigcup_{x \in \mathbb{R}^{2} \backslash I^{2}} B_{2}(x) .
$$

Базис $B$ определим следующим образом:

$$
B(x)=\left\{I: I \ni x, I \in R_{1} \cup R_{2} \cup R_{3}\right\}, \quad x \in \mathbb{R}^{2} .
$$

Пусть

$$
x \in A \backslash \varlimsup_{k \rightarrow \infty} G_{k} .
$$

Нетрудно убедиться, что $B(x)$ представляется в виде $B(x)=\left\{I: I \ni x, I \in R_{1}\right\} \cup T$, где $T$ - семейство интервалов с диаметрами, превьшаюшими некоторое положительное число. Рассмотрим произвольную последовательность $\left\{I_{m}\right\} \subset B(x), \operatorname{diam} I_{m} \rightarrow 0$, $m \rightarrow \infty$. Тогда для достаточно большого $m_{0}$ имеем $I_{m} \in R_{1}$ при $m>m_{0}$. Следовательно, найдутся натуральные числа $k_{m}\left(m>m_{0}\right)$ такие, что $I_{m} \in T_{k_{m}}\left(m>m_{0}\right)$. Отсюда, учитывая (15), получаем

$$
\frac{1}{\left|I_{m}\right|} \int_{I_{m}} f \geqslant k_{m}, \quad m>m_{0}
$$

Нетрудно видеть, что $k_{m} \rightarrow \infty$ при $m \rightarrow \infty$. Отсюда имеем

$$
\underline{D}_{B}\left(\int f, x\right)=\infty .
$$

Очевидно, $\varlimsup_{k \rightarrow \infty} G_{k}$ имеет меру нуль. Непосредственно из последнего равенства заключаем, что

$$
f(x)<\underline{D}_{B}\left(\int f, x\right)=\infty
$$

почти всюду на $I^{2}$. Теорема 2 доказана. 
ЗАмЕчАниЕ 1. Если $B \subset B_{2}-$ ТІ-базис, то для каждой $f \in L\left(\mathbb{R}^{2}\right)$

$$
\underline{D}_{B}\left(\int f, x\right) \leqslant f(x) \leqslant \bar{D}_{B}\left(\int f, x\right)
$$

почти всюду на $\mathbb{R}^{2}$. Действительно, найдется ТІ-базис $B^{\prime} \subset B$, для которого $B^{\prime}(0)=$ $\left\{I_{k}\right\}$, где $I_{k} \supset I_{k+1}, k \in \mathbb{N}$, и $\operatorname{diam} I_{k} \rightarrow 0$ при $k \rightarrow \infty$. Используя лемму Гусмана (см. [2, c. 14]), с помощю известной стандартной техники (см., например, [2, с. 36]) можно доказать, что $B^{\prime}$ дифференцирует $\int f$ для каждой $f \in L\left(\mathbb{R}^{2}\right)$. Следовательно, для любой $f \in L\left(\mathbb{R}^{2}\right)$

$$
\underline{D}_{B}\left(\int f, x\right) \leqslant \underline{D}_{B^{\prime}}\left(\int f, x\right)=f(x)=\bar{D}_{B^{\prime}}\left(\int f, x\right) \leqslant \bar{D}_{B}\left(\int f, x\right)
$$

почти всюду на $\mathbb{R}^{2}$.

ЗАмечание 2 . Пусть $B \subset B_{2}$ - ТІ-базис и $f \in L\left(\mathbb{R}^{2}\right), f \geqslant 0$. Учитьвая замечание 1 , имеем $0 \leqslant \underline{D}_{B_{2}}\left(\int f, x\right) \leqslant \underline{D}_{B}\left(\int f, x\right) \leqslant f(x)$ почти всюду на $\mathbb{R}^{2}$. Отсюда в силу теоремы Безиковича заключаем, что почти всюду на $\mathbb{R}^{2} \underline{D}_{B}\left(\int f, x\right)=f(x)$. Следовательно, последнее равенство справедливо почти всюду на $\mathbb{R}^{2}$ для базиса $B$ и функции $f$ из теоремы 1.

ЗАмЕчАНИЕ 3. Предположим, что последовательность $h_{k} \uparrow \infty, h_{k}>1$, кроме (2) удовлетворяет еще и неравенству $\sum_{k=1}^{\infty} 1 / h_{k}<1 / 9$. Тогда, рассматривая функцию $f=\sum_{k=1}^{\infty}(-1)^{k} f_{k}$, где $f_{k}, k \in \mathbb{N},-$ функции из доказательства теоремы 1, с помощью оценок, установленных при доказательстве теоремы 1 , убеждаемся, что

$$
f(x)<\bar{D}_{B}\left(\int f, x\right)<\infty \quad \text { и } \quad-\infty<\underline{D}_{B}\left(\int f, x\right)<f(x)
$$

почти всюду на $I^{2}$.

\section{СПИСОК ЦИТИРОВАННОЙ ЛИТЕРАТУРЫ}

[1] Besicovitch A.S. On differentiation of Lebesgue double integrals // Fund. Math. 1935. V. 25. P. 209-216.

[2] Гусман М. Дифференцирование интегралов в $\mathbb{R}^{n}$. М.: Мир, 1978. 